\title{
OVERLAPPING GRIDS FOR THE SIMULATION OF PARTICLE INTERACTION AT THE MICRO SCALE
}

\author{
Hermann Nirschl \\ Institute for Mechanical Process Engineering, University of Karlsruhe (TH), Germany \\ E-mail: Hermann.Nirschl@mvm.uni-karlsruhe.de
}

\begin{abstract}
This paper describes the use of overlapping grids for the calculation of flow around single and multipleparticle configurations at the micro scale. The basic equations for calculation are those for conservation of mass and momentum which are solved using a common Finite-Volume formulation. The hydrodynamic particle-particle and particle-wall interaction can be calculated by using an overlapping or Chimera grid scheme. With the grid structuring procedure it is possible to use simple and structured grids around the particles and the overall main grid geometry. The particle grids are lapped over the main grid such that they can move independently after each time step without remeshing the whole geometry. The paper gives results for the validation of the code developed for general test cases, for a rotating ellipsoid in simple shear flow, the flow around particles attached to a wall, the motion of a particle in the vicinity of a wall and some results for the flow through a packed bed configuration.
\end{abstract}

Keywords particle flows, numerical simulation, overlapping grids, particle-wall interaction, packed beds

\section{Introduction}

Particles play an important role in a lot of engineering processes. Particle motion in fluidised beds, filtration, separation and sedimentation are just a few examples where particulate systems are treated for the generation of a special product design. The processes depend very strongly on the material properties of the particle systems which are very hard to implement into a general description for the particle motion. But also for simple or test cases, the mass and momentum transfer in the dense systems are very challenging for simulation. Nowadays the available computer resources are, however, still limited to simulate the whole process at the micro scale. For the macro scale there are methods available but in general they depend on empirical coefficients and factors which describe the physical properties at the micro scale. The macro schemes in general describe the particles as a second phase which interacts with the fluid phase.

The details of the flow can just be resolved at the micro scale. Analytic results with simple general equations are available for low Reynolds numbers and with limited number of particles. Numerical methods are now very common to overcome these limitations, but for the difficult geometries which can result in particle configurations the generation of the grids is very time consuming because of remeshing after each time step when the particles have changed their locations.

This limitation can be solved by overlapping meshes where each particle has its own spherical grid. For the case of a non-spherical particle the grid can also be easily readjusted. The single particle grids are then overlapped over a main mesh which describes the major geometry. The information between the two grids is interchanged at the so-called fringe points. The variables at the fringe points are interpolated from the main or the minor meshes respectively. The disadvantage of this scheme is that the number of particles which can be handled is very limited. The scheme is available for the calculation of the flow around particle configurations where the particle-particle or particle-wall interaction can be resolved in detail.

\section{Governing Equations and Grid Scheme}

The flow around particles is calculated by solving the continuity and momentum equations in their integral and conservative forms:

$$
\begin{gathered}
\iint \vec{v} \cdot \mathrm{d} \vec{A}=0, \\
\frac{\partial}{\partial t} \iiint \vec{V} \mathrm{~d} V+\iiint \vec{V} \cdot \nabla \vec{v} \mathrm{~d} V=-\iint p \mathrm{~d} \vec{A}+\frac{1}{R e} \iint \nabla \vec{V} \mathrm{~d} \vec{A} .
\end{gathered}
$$

These equations are formulated in the dimensionless form with $\vec{v}$ as the velocity vector and $p$ as the dynamic pressure. $\mathrm{d} V$ is a discrete volume and $\mathrm{d} \vec{A}$ is the discrete area vector in the Finite-Volume formulation. The Reynolds number $R e$ is defined as follows:

$$
R e=\frac{U D}{v},
$$

where $U$ is the undisturbed fluid velocity, $D$ the characteristic diameter of the particle and $v$ the kinematic viscosity.

The equation of motion was solved according the low Mach number approximation with Chorin's projection scheme (Chorin, 1967) where the momentum equation is linearized and solved first implicitly for the velocity with the old pressure field. In a second step, a Poisson equation, derived from the continuity equation, is iterated until convergence is achieved for the continuity (Nirschl et al., 1995). With this result the pressure can be corrected in the momentum formulation. The whole scheme is of second-order accuracy in space and time. The disadvantage of the scheme is the relatively high number of iterations which is necessary to converge the Poisson equation. For general test cases, around 20 iterations are necessary. 
The geometry of the particles is described by a curvilinear coordinate system which is adapted to the particle shape. As already mentioned in the introduction, an overlapping grid scheme was used for the calculation of particle-wall or particle-particle interactions. The basic idea of the method is that the spherical grid which describes the geometry of the particle is overlapped over a major grid which is used for the description of the whole overall geometry (see Fig.1). Information is interchanged at the so-called 'fringe points', shown in the figure as filled squares. At those points the velocity vector or the pressure is interpolated from the minor or major mesh, respectively. At the outer points of the minor particle mesh the information is interpolated from the major mesh. The points of the major mesh close to the surface of the rigid particle are interpolated from the minor grid. The hollow points, i.e. the points of the major mesh where the rigid particle is located are the so called 'holes'. The points of the minor mesh which are outside the major geometry are also treated as holes. Those points in the solution matrix are set to zero. Care should be taken that the fluxes entering and leaving the boundaries of the minor or major mesh be balanced. Otherwise the continuity equation at the boundaries would not be fulfilled leading to pressure oscillations for the otherwise incompressible flow conditions. For interpolation, a linear procedure was chosen. After some testing with higher-order schemes, it could been shown that efforts for a higher-order accuracy scheme is not necessary for calculations in the low- and medium-Reynolds number range.

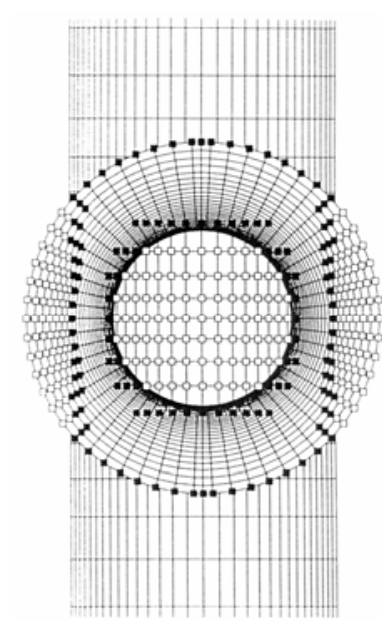

Fig. 1 Spherical mesh lapped over a rectangular major geometry: holes are marked with hollow squares and fringe points with filled squares.

The major advantage of this overlapping grid scheme is that the minor mesh can be easily moved relative to the major mesh. This means that particle motion can be calculated without remeshing the whole geometry. Also the calculation and the behaviour of a particle which is close to a rigid wall can be easily investigated. The points of the minor mesh of a particle which are within the rigid wall are treated as holes, so that they are eliminated from the flow calculation. When the particle moves to another location it can retain its mesh, and only the holes and fringe points need to be defined again.

Another advantage of the scheme is the treatment of not just one single particle mesh but also multiple meshes (Fig. 2), that is, several spherical meshes can be lapped over the major mesh. It is also possible to overlap single minor meshes and calculate the fringe points in between the minor meshes. The particle meshes can be treated independently of each other.

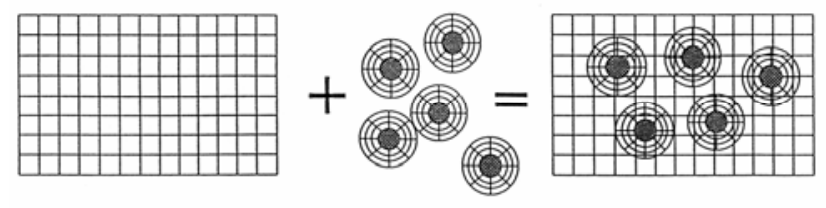

Fig. 2 Multiple minor meshes around spherical particles are lapped over a rectangular major geometry.

The whole code and the overlapping grid procedure have been verified for different two- and three-dimensional test cases to compare pressure and shear stress distributions of the particle surface as well as drag coefficients. Fig. 3 shows the drag coefficient $c_{d}$ as a function of Reynolds number $R e$ for uniform flow around a single sphere in the low- and medium-Re range. Comparison with data (Clift et al., 1978) from the literature for this test case is also excellent.

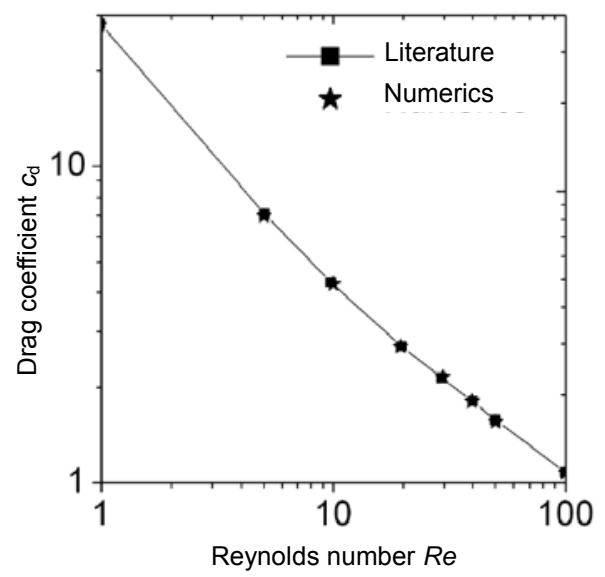

Fig. 3 Calculated drag coefficient $c_{d}$ versus Reynolds number Re for the simulation of uniform flow around a single sphere with an overlapping grid configuration.

\section{Results and Discussion}

The following are problems of fundamental interest in particle-laden flows in engineering applications as well as in rheology.

\subsection{Motion of a rotating ellipsoid in simple shear flow}

It is well known that spherical particles move with constant angular velocity while the motion of non spherical 
particles is strongly time-dependent. The classical work on the analytical description of the motion of an ellipsoidal particle in viscous flow by Jeffery (1922/23) will be extended into the medium Reynolds number range by means of numerical methods. The ellipsoidal particle is supposed to move without any relative velocity to undisturbed simple shear flow which can be characterized by a constant shear rate. The particle will then rotate about the $y$-axis in the $x z$-plane with a time dependent angular velocity (Fig.4).

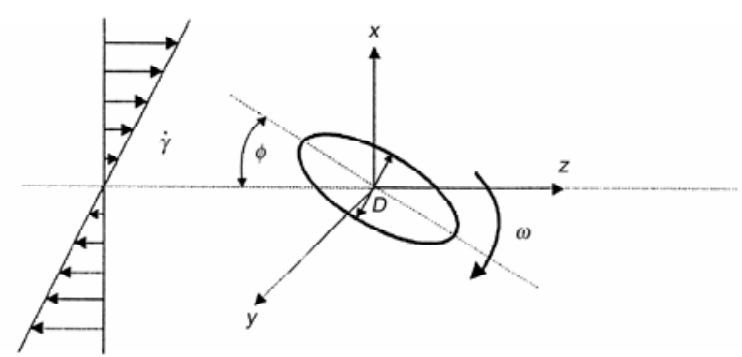

Fig. 4 General configuration for calculation of a rotating ellipsoidal particle in simple shear flow.

The major mesh is rectangular and an elliptic minor mesh describes the particle shape by means of curvilinear coordinates. Calculations in the minor mesh have been performed in a rotating frame of reference at the midpoint with the angular velocity of the particle.

Fig. 5 shows the distribution of the torque coefficients over the angle of attack $\beta$ for an ellipsoid with an axis ratio of $\alpha=2$ (quotient of the major to the minor axis) and at a Reynolds number of $R e=0.1$. At $\beta=0^{\circ}$, where the major axis of the particle is parallel to the $z$-coordinate, we find a positive shear torque and a negative pressure torque. The shear forces tend to move the particle clockwise while pressure torque acts counter clockwise thus balancing the torques with each other. With increasing angle of attack the pressure becomes dominant and speeds up the particle until the major axis is parallel with the $x$-axis of the computational domain. At higher angles of attack the pressure torque influence decreases while the shear stress dominates the particle motion. The particle motion is decelerated because of the relatively high shear forces between the rotating particle and the fluid until the forces balance each other again at $\beta=180^{\circ}$.

Higher axis ratios lead to a decrease of the angular velocity at $\beta=0^{\circ}$ when the major axis is parallel to the $z$-axis. Thus the particle becomes more and more aligned in the flow with increasing axis ratio because of the increasing moment of inertia. Fig. 6 gives the time $T$ which is necessary for one rotation over the axis ratio $\alpha$ for $R e=0.1$ and $R e=1$. The analytical solution given by Jeffery shown in Fig. 6 for pure viscous particle motion indicates that the rotation time $T$ depends almost linearly upon the axis ratio $\alpha$. For small axis ratios the Reynolds number influence is negligible while with increasing Reynolds number and axis ratio the relationship becomes more and more non linear.
The numerical solution for $R e=0.1$ is relatively close to Jeffery's solution. However for $R e=1$ the differences are very significant. This means that the particles remain much longer in the region around $\beta=0^{\circ}$ where the particle is aligned in the flow: The reason for this behaviour is that the pressure and shear stress distributions over the particle surface have changed so much at higher Reynolds numbers that the viscous shear stresses at the particle surface are not high enough to rotate the particle away from its equilibrium position.

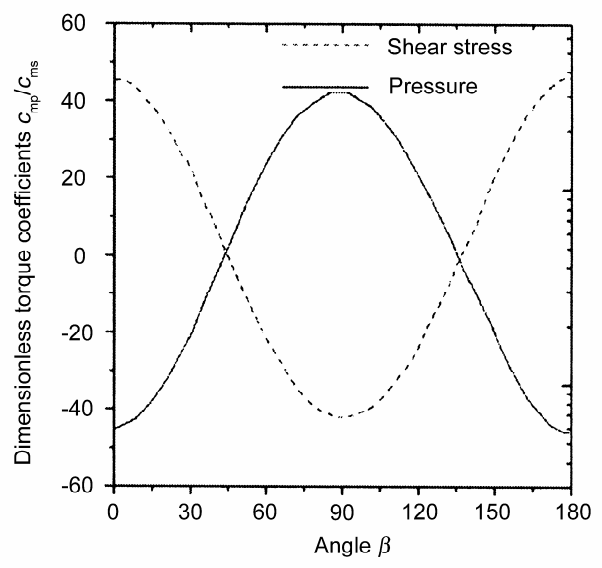

Fig. 5 Shear and pressure torque coefficients $c_{\mathrm{ms}}$ and $c_{\mathrm{mp}}$ versus the angle of attack $\beta$ for an ellipsoid with an axis ratio of 2 .

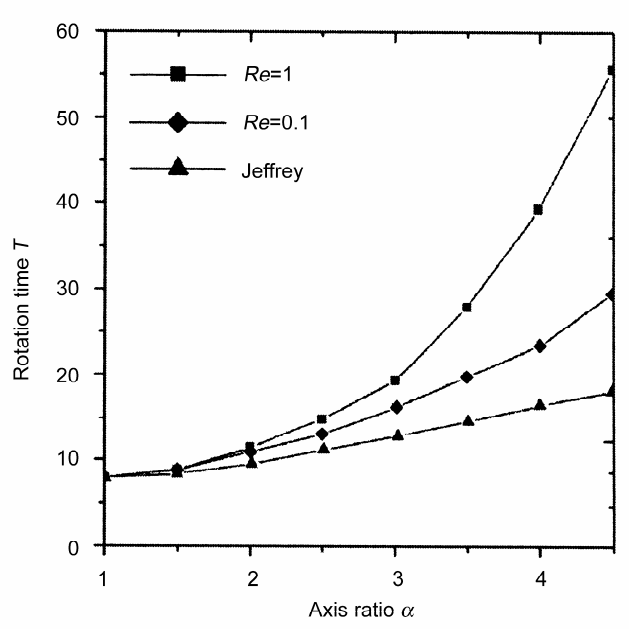

Fig. 6 Rotational time $T$ versus axis ratio $\alpha$ for different Reynolds numbers: comparison with analytic results.

At a Reynolds number of $R e=10$ we have observed two possible behaviours of the particle depending on the initial conditions: either (i) the rotation time goes to infinity and no periodic motion can be detected with the particle moving to a stable position, or (ii) the particle moves with a rotation time which is less than the rotation time in solutions for low Reynolds number. With further increasing axis ratio, the rotation time does not change significantly. Fig. 7 shows the two possible configurations of the ellipsoid depending on the axis ratio. 


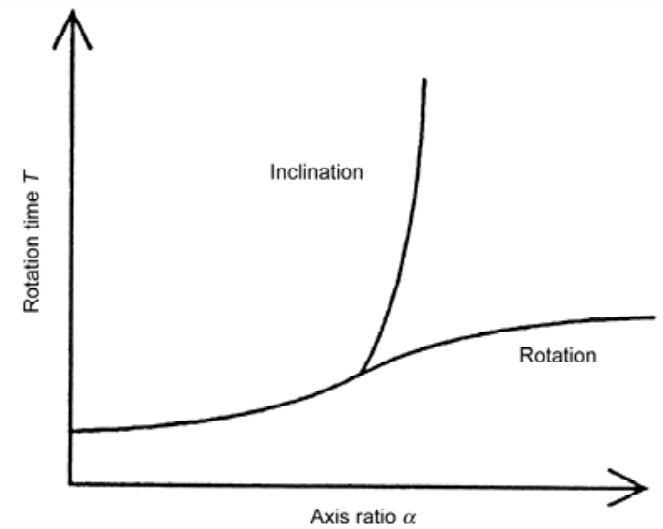

Fig.7 Bifurcation for an ellipsoidal particle at Reynolds number $R e=10$.

It seems that a bifurcation exists where either inclination or rotation is possible. The particle motion strongly depends on the way the motion was first started. For low Reynolds numbers $(R e \approx 1)$ the starting conditions have no influence and the particle will always go to a stable position. For a Reynolds number with sufficient influence of inertia $(R e \approx 10)$ the motion depends on the axis ratio. The area where we get the inclination at a special angle of attack increases with growing aspect ratio. With increasing axis ratio the angle of attack increases until it stays constant. From the theory of bifurcations we can conclude that this behaviour can be described by the so-called 'saddle-node bifurcation'.

\subsection{Flow around a single particle in a laminar boundary layer}

Fig. 8 shows schematically the linear shear flow around a rigid particle with diameter $D$ at a fixed distance $h$ from a rigid wall. The curvilinear coordinate system $(\xi, \eta, \zeta)$ describes the grid for calculating the velocity field in the boundary layer, and the curvilinear coordinate system $\left(\xi_{1}, \eta_{1}, \zeta_{1}\right)$ the geometry of the particle. The Reynolds number is calculated from the undisturbed fluid velocity along the particle axis $U$. As an additional parameter for the description we use the distance of the particle to the rigid wall $h$, which is related to the particle diameter and has therefore no dimension. The shear rate can be calculated from the undisturbed fluid velocity and the wall distance. It should be mentioned that for this type of calculation some parts of the minor geometry are located within the rigid wall. This means that additional holes and fringe points have to be introduced. All the points within the rigid wall are handled as holes. Additional fringe points for the minor geometry have to be added to interpolate the points close to the wall from the major rectangular geometry.

This problem can be regarded as a general test case for the calculation of particle-wall interaction because experimental values are available (Rubin, 1977). For practical cases the particles will move along the rigid wall. This case will be regarded further on in this paper.

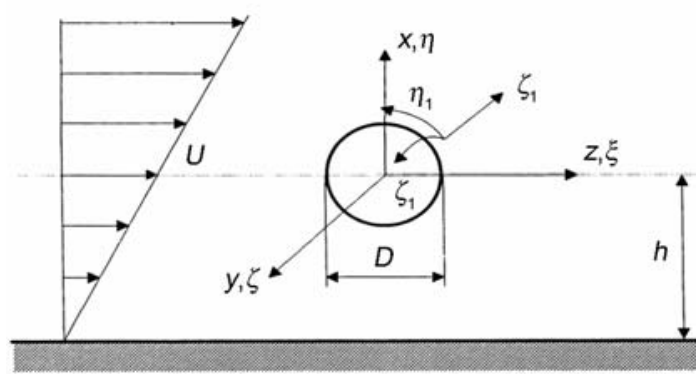

Fig. 8 Schematic description of the geometry for calculating the flow around a spherical particle close to a rigid wall.

For this discussion we first take a look at the drag and lift coefficients. For the drag coefficient $c_{d}$ the whole viscous forces and pressures are integrated over the geometry in the undisturbed flow direction. For the lift coefficient $c_{1}$ the integration is perpendicular to it. Both coefficients consist of two parts: a value coming from the pressure $\left(c_{\mathrm{dp}}, c_{\mathrm{lp}}\right)$ and one coming from the shear stresses $\left(c_{\mathrm{ds}}, c_{\mid \mathrm{s}}\right)$. The values are calculated in the usual way where the forces are related to the stagnation pressure and the projection area of the particle:

$$
\begin{aligned}
& c_{\mathrm{d}}=c_{\mathrm{dp}}+c_{\mathrm{ds}}=-\frac{\iint p \vec{e}_{z} \cdot \mathrm{d} \vec{A}}{1 / 2 \rho U^{2} D^{2} \pi / 4}+\frac{\iint \vec{e}_{z} \cdot \bar{\tau} \cdot \mathrm{d} \vec{A}}{1 / 2 \rho U^{2} D^{2} \pi / 4}, \\
& c_{1}=c_{\mathrm{lp}}+c_{\mathrm{ls}}=-\frac{\iint p \vec{e}_{x} \cdot \mathrm{d} \vec{A}}{1 / 2 \rho U^{2} D^{2} \pi / 4}+\frac{\iint \vec{e}_{x} \cdot \tau \cdot \mathrm{d} \vec{A}}{1 / 2 \rho U^{2} D^{2} \pi / 4},
\end{aligned}
$$

where the vector $\vec{e}$ gives the unit vector in the three directions of the coordinate system.

Fig. 9 shows the drag values versus the wall distance and the Reynolds number as a parameter. With a constant Reynolds number and decreasing wall distance an increasing drag coefficient can be detected. With increasing

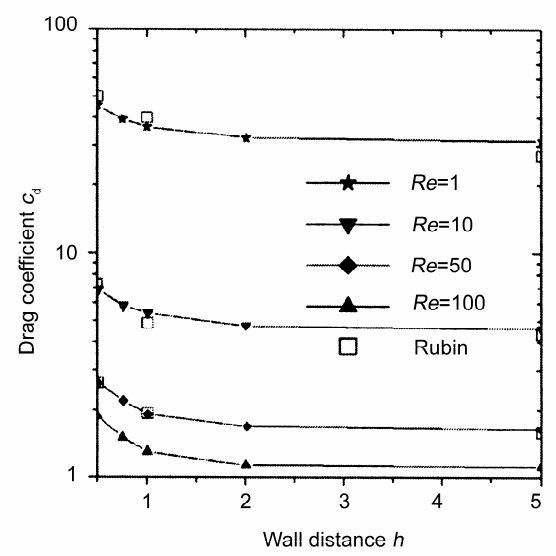

Fig. 9 Drag coefficient $c_{d}$ for flow around a particle close to a rigid wall for different Reynolds numbers: comparison with Rubin's experimental data. 
Reynolds number this effect is even more pronounced because of separation effects in the wake of the particle arising from interaction with the wall. The increasing drag coefficient is also a direct effect of the wall influence, that is, it is not possible for the fluid to move in the gap between particle and wall, and the fluid is forced to flow over and around the particle. For increased distance to the wall the drag values approach more and more those for uniform flow. It can be noted that when the distance is larger than $h>2$, the wall loses its influence on the drag. The figure also shows that the maximal deviation from the experimental values of Rubin (1977) is below $5 \%$.

Fig. 10 shows the variation of the lift coefficient with the above mentioned parameters: increasing, in the same way as the drag coefficient, with decreasing Reynolds number. For low Reynolds numbers the influence of the close wall is more pronounced than that for the drag coefficient. The wall influence on the lift coefficient is superimposed by the lift coefficient in a linear shear field where the wall is far away from the particle surface. For low Reynolds numbers it was shown that the lift coefficient is linearly dependent on the shear rate. In the curves of the figure the non linear part comes therefore directly from the wall influence.

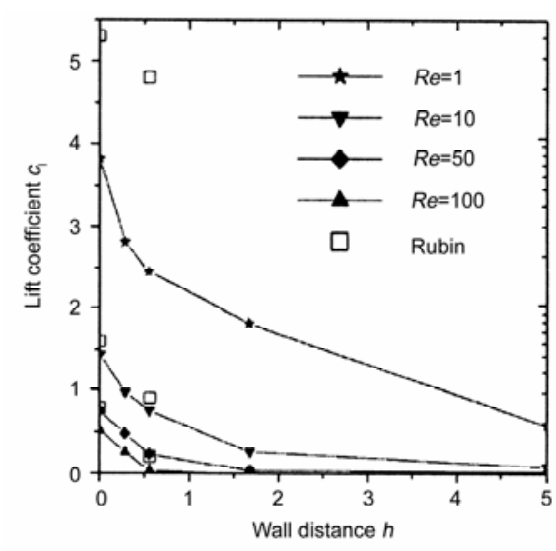

Fig. 10 Lift coefficient $c_{\text {| }}$ for flow around a particle close to a rigid wall for different Reynolds numbers: comparison with Rubin's experimental data.

In all the calculations the lift forces are always positive, meaning that in all real flow a particle always tends to move away from the wall. Comparison with measurements again shows that deviations between numerical results and experimental values are relatively low except for values for the Reynolds number of $R e=1$. There are different reasons for this. The experiments were performed in tube flow with a parabolic velocity profile with a ratio of the particle diameter to the tube diameter of 1:25. For very low Reynolds numbers it is well known that the wall influence is significant on such flow over the particle. In the numerical simulations a linear shear field was selected as the initial and undisturbed flow. Moreover it should be mentioned that with decreasing Reynolds number the ratio between the lift to the drag coefficient $c_{1} / c_{d}$ decreases, implying that with decreasing Reynolds number the accuracy for both the calculation and the experiments diminishes.

\subsection{Flow around a single particle attached to a rigid wall}

The flow around a spherical as well as an ellipsoidal particle at different angles of attack attached to the rigid wall are studied in the following investigation. The ellipsoid has again an axis ratio of 2 and is located at the wall at an angle of attack of $0^{\circ}$ and $90^{\circ}$. For the calculation of the Reynolds number the undisturbed fluid velocity at $0.5^{\star} \mathrm{D}$ distance to the wall was chosen. The undisturbed fluid profile has a constant velocity gradient. This approximation should be valid for cases, when the particle diameter is negligible compared to the characteristic length of the major flow. But in principle it is possible to use any laminar flow profile as the initial condition.

For the overlapping grid scheme considered, a part of the minor mesh is again located outside the computational domain in the rigid wall. Those points are treated as holes. The points in the minor mesh which are close to the wall are interpolated as fringe points from the major mesh.

Fig. 11 shows the drag coefficient $c_{d}$ versus the Reynolds number $R e$ for both the small and intermediate Reynolds number regimes. The curves are linear in the low Reynolds number range, becoming non linear with increasing Reynolds number, primarily due to the increasing influence of inertia which leads to separation at Reynolds numbers higher than $R e=20$. For the ellipsoid at $\beta=90^{\circ}$ and Reynolds numbers $R e>80$ non-stationary separation could be observed. Besides, for both particles, a horseshoe vortex was detected in the frontal area where the flow approached the surface of the particle.

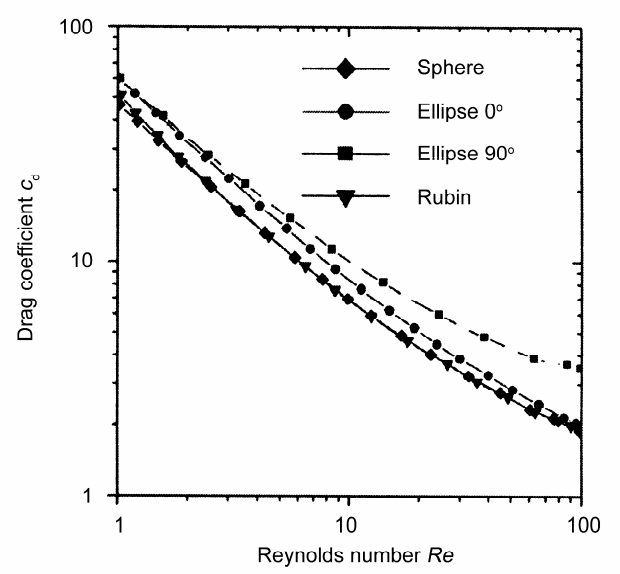

Fig. 11 Drag coefficient $c_{d}$ versus Reynolds number $R e$ for a sphere and an ellipsoid at different angles of attack.

The drag values for the ellipsoids are always significantly higher than those for the spheres, primarily because of the larger surface of the ellipsoidal particles. At high angle of 
attack, separation effects have an additional influence on increasing the pressure drop over the surface from the front to the rear.

For this configuration it is also possible to detect lift coefficients for the particle due to lift forces which arise through amplification of the pressure minimum at the upper side. The lift forces have an increasing importance at higher Reynolds numbers.

Fig. 12 shows the variation of the lift coefficient $c_{1}$ with the Reynolds number $R e$, linear for small $R e$ and non linear at large $R e$ specially for ellipsoids. The reason for this is again the ongoing separation on the particle surface which leads to a strong pressure minimum. The ratio between lift to drag coefficients $c_{1} / c_{d}$ is app. 0.002 for $R e=0.01$ and 0.3 for $R e=100$. This means that for Stokes flow practically no lift can be detected because of the elliptic behaviour of the governing equations.

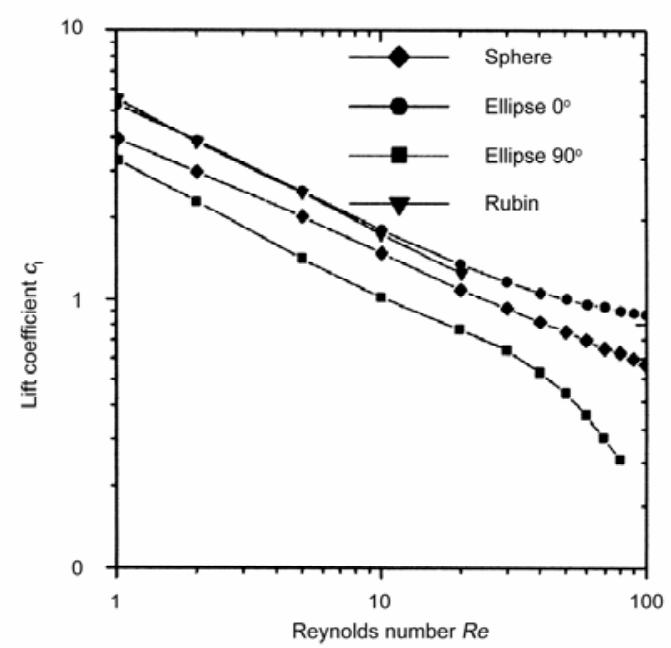

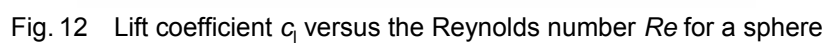
and an ellipsoid at different angles of attack.

Rubin (1977) determined the drag and lift coefficients for a single sphere in laminar flow close to the wall of a tube which fit very well with the calculated values, as are shown in Figs. 11 and 12. Slight differences for the lift coefficients may again be due to the parabolic profile. The lift forces are in general relatively low compared to the calculated and measured drag forces. For the calculated values the accuracy depending on discretization is between $1 \%$ and 3\% (Nirschl \& Polzer, 1996).

\subsection{Fluid dynamic behaviour of a moving single particle close to a rigid wall}

The behaviour and motion of a single particle close to a rigid wall will next be examined. The particle moves with the undisturbed fluid at a distance $h$ relative to the rigid wall in a linear shear field. The particle rotates in the shear field according to the pressure and shear forces acting on the particles surface. In undisturbed shear flow without any wall influence the particle would rotate for a Reynolds number approaching zero with $\omega=0.5 \dot{\gamma} \mathrm{s}^{-1}$. Due to wall influence, the lift coefficient as well as the angular velocity depends on the wall distance as well as the Reynolds number. The angular velocity was adjusted after each time step according to the calculated angular momentum, so that the particle could rotate freely in the flow. Fig. 13 shows the dependence of the lift coefficient on the Reynolds number with the wall distance $h$ as a parameter (for $h=0.5$ the particle is located directly at the wall). Far away from the wall there is no detectable lift. At small distances to the wall there is detectable lift over the whole Reynolds number region investigated. Especially for low Reynolds numbers, the influence of the wall on the flow around the rotating particle is highly significant because of the strongly diffusive character of the flow. In general, the wall plays a leading role in influencing the lift coefficient on the particle, dropping to insignificance for wall distances $h>3$, that is, as far as the accuracy of the calculations could detect.

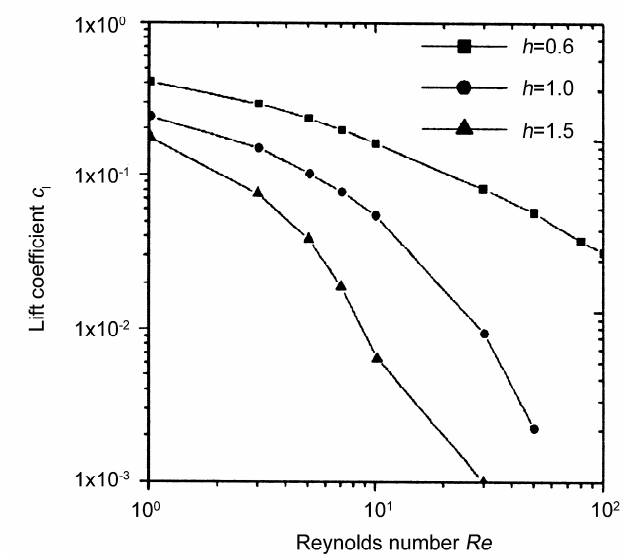

Fig. 13 Lift coefficient $c_{\text {| }}$ versus the Reynolds number $R e$ for different wall distances.

\subsection{Numerical simulation of local momentum transfer in packed beds}

As a final example which shows the possibilities for the present analysis of flow structures at the micro scale using the overlapping grid approach, the local momentum transfer in a packed bed will be considered. The literature abounds with experimental investigations in progress on the distribution of velocities, pressure drop and heat transfer at different Reynolds numbers for different configurations of packed beds (e.g., Vortmeyer \& Schuster, 1983; Eigenberger \& Bey, 1996). With the proposed numerical scheme it is possible to resolve the structure of three-dimensional flow around 300 randomly distributed particles in a cylindrical tube. It is of major interest to understand the transport properties close to the rigid tube wall.

Each sphere of the configuration has again its own spherical mesh. All minor meshes overlap each other to 
form a configuration which is overlapped with the major grid to describe the tube geometry. As a further development of the program code, the spherical meshes for the particles have been modified such that each outer boundary of a minor mesh is adjusted to match the surface of the neighbouring particles. This has the advantage that no uncalculated holes would be lost in the simulation. The points of contact between the particles have thus been fixed as rigid 'connecting points', meaning that they are handled as holes and the equations are not to be solved there. The random distribution of the particles in the cylindrical tube was designed according to the algorithm of Chan \& Ng (1986).

Many correlations are available in the literature for calculating pressure drop in packed beds. The code was validated with the classical approximations by Darcy (1856), Ergun (1952) and Molerus (1977). The agreement between the numerical results and the analytical expression is quite well (Debus, 1998; Debus et al., 1998).

Fig. 14 shows the velocity distribution in the packed bed. The isolines on the particle surface represent the pressure distribution within the bed. The Reynolds number based on the diameter of the tube for this calculation is $R e=23$. In the areas close to the tube wall the porosity of the packed bed is relatively high as compared to those in the inner regions of the tube. The fluid drag along the tubular wall is therefore less than in the inner regions, thus leading to relatively high axial velocities. An overshooting can be observed which has the technical disadvantage that the residence time distribution for the fluid may change by as much as one order of magnitude in the radial direction. A detailed analysis of the data files also shows that wakes and recirculating flows can be observed for the Reynolds numbers and porosities chosen with additional negative influences on the residence time distribution.

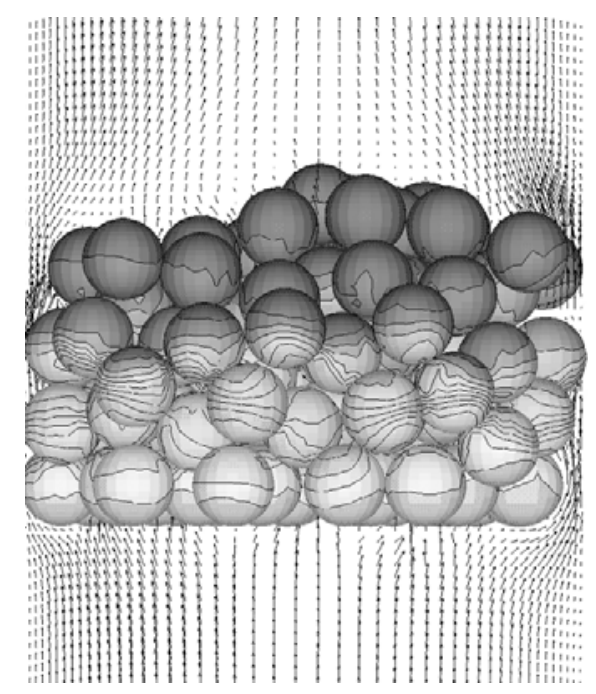

Fig. 14 Velocity vectors for flow through a packed bed at $R e=23$ for flow coming from the bottom, with particles located on a virtual membrane.
The stream lines, which are strongly bent around the particles, compressing and elongating the fluid elements during their passage through the packed bed, provide a helpful depiction of the flow physics among the particles. For Newtonian fluids this has no effect, but for viscoelastic media it will have a significant influence on pressure drop.

\section{Summary and Conclusions}

- An overlapping grid scheme approach is presented for calculating particle-laden flows which shows specific advantages as compared to other grid generation schemes.

- The code was validated and compared with available experimental data from the literature.

- Specifically, the scheme is very helpful to analyse and understand the flow physics of particle motion, particle-wall and particle-particle interactions. In detail, the values for drag, lift and torque can be calculated and help to realize approximations for micro scale models which are necessary for the development of computer codes for the calculation of macro scale flow structures.

- The numerical approach presented is however limited in the number of particles under calculation, though the code can handle as many as hundreds of particles as long as they are fixed in location. The method is a challenge for the future, not only to depict the mechanical particle-particle interactions but also to understand the dynamic behaviour concerning such interactions. There is still a long way to go, although increasing computer resources and the emerging massively parallel systems will help us to close the gap between the micro and the macro approaches.

\section{Nomenclature}

$\begin{array}{ll}C_{\mathrm{d}} & \text { drag coefficient } \\ C_{\mathrm{dp}} & \text { drag coefficient, pressure part } \\ C_{\mathrm{ds}} & \text { drag coefficient, shear stress part } \\ C_{\mathrm{I}} & \text { lift coefficient } \\ C_{\mathrm{lp}} & \text { lift coefficient, pressure part } \\ C_{\mathrm{ls}} & \text { lift coefficient, shear stress part } \\ C_{\mathrm{mp}} & \text { torque coefficient, pressure part } \\ C_{\mathrm{ms}} & \text { torque coefficient, shear stress part } \\ D & \text { particle diameter, } \mathrm{m} \\ \vec{e} & \text { unity vector } \\ \mathrm{d} \vec{A} & \text { area vector } \\ \mathrm{d} V & \text { discrete volume } \\ h & \text { wall distance } \\ p & \text { hydrodynamic pressure } \\ R e & \text { Reynolds number } \\ T & \text { rotation time, } \mathrm{s} \\ U & \text { undisturbed fluid velocity, } \mathrm{m} \cdot \mathrm{s}^{-1}\end{array}$




$\begin{array}{ll}\vec{v} & \text { velocity vector } \\ \alpha & \text { axis ratio of an ellipsoidal particle } \\ \beta & \text { angle of attack, rad } \\ \dot{\gamma} & \text { shear rate, } \mathrm{s}^{-1} \\ v & \text { kinematic viscosity, } \mathrm{m} \cdot \mathrm{s}^{-2} \\ \xi, \eta, \zeta & \text { curvilinear coordinates } \\ \omega & \text { angular velocity, } \mathrm{s}^{-1}\end{array}$

\section{References}

Chan, S. \& Ng, K. (1986). Geometrical characteristics of a computer-generated three-dimensional packed column of equal and unequal sized sphere with special reference to wall effects. Chem. Eng. Commun., 48, 215-236.

Chorin, A. (1967). A numerical method for solving incompressible viscous flow problems. J. Comput. Phys., 2, 12-26.

Clift, R., Grace, J. R. \& Weber, M. E. (1978). Bubbles, Drops and Particles. Academic Press.

Darcy, H. P. G. (1856). Les Fontaines Publiques de la Ville de Dijon. Paris: Victor Dalmont.

Debus, K. (1998). Numerische Untersuchung des Kugelhaufendurchströmung —Ansätze zur Berechnung strömungsbedingter Deformationen verformbarer Körper. PhD thesis, TU-München.
Debus, K., Nirschl, H., Delgado, A. \& Denk, V. (1998). Numerische Simulation des lokalen Impulsaustausches in Kugelschüttungen. Chem. Ing. Tech., 70, 415-418.

Eigenberger, G. \& Bey, O. (1996). Bestimmung von Strömungsverteilung und Wärmetransportparametern in schüttungsgefüllten Rohren. Chem. Ing. Tech., 68, 1294-1299.

Ergun, S. (1952). Fluid flow through packed columns. Chem. Eng. Prog., 48, 89-94.

Jeffery, G. B. (1922/23). The motion of ellipsoidal particles immersed in a viscous fluid. Proc. R. Soc. London, Ser. A, 102.

Molerus, O. (1977). Druckverlustgleichung für die Durchströmung von Kugelschüttungen im laminaren und im Übergangsbereich. Chem. Ing. Tech., 49, 675.

Nirschl, H., Dwyer, H. A. \& Denk, V. (1995). Three-dimensional calculations of the simple shear flow around a single particle between two moving walls. J. Fluid Mech., 283, 273-285.

Nirschl, H. \& Polzer, S. (1996). Strömungstechnisches Verhalten einzelner Partikel in wandnahen, laminaren Grenzschichten. Chem. Ing. Tech., 68, 409-412.

Rubin, G. (1977). PhD thesis, University of Karlsruhe.

Vortmeyer, D. \& Schuster, H. (1983). Evaluation of steady flow profiles in rectangular and circular packed beds by variational methods. Chem. Eng. Sci., 38, 1691-1699.

Manuscript received February 16, 2005 and accepted March 7, 2005.

\title{
DRAG ON SUBMICRON NANOPARTICLE AGGREGATES
}

\author{
F. Einar Kruis \\ Faculty of Engineering Sciences, University of Duisburg-Essen, Campus Duisburg, Bismarckstraße 81, 47057 Duisburg, Germany \\ E-mail: e.kruis@uni-duisburg.de
}

\begin{abstract}
A new procedure was developed for estimating the effective collision diameter of an aggregate composed of primary particles of any size. The coagulation coefficient of two oppositely charged particles was measured experimentally and compared with classic Fuchs theory, including a new method to account for particle non-sphericity. A second set of experiments were performed on well-defined nanoparticle aggregates at different stages of sintering, i.e. from the aggregate to the fully sintered stage. Here, electrical mobility was used to characterize the particle drag. The aggregates are being built from two different size-fractionated nanoparticle aerosols, the non-aggregated particles are discarded by an electrofilter and then they are passed through a furnace at concentrations low enough not to induce coagulation.
\end{abstract}

